\title{
Correlation and Path Coefficient Analysis in Chilli (Capsicum annuum L.) for Yield and Yield Attributing Traits
}

\author{
Manoj Kumar Bundela ${ }^{1 *}$, S.C. Pant ${ }^{1}$, Madhuri ${ }^{2}$ and Kulveer Singh ${ }^{3}$ \\ ${ }^{1}$ Department of vegetable science, College of Horticulture, V.C.S.G Uttarakhand University of \\ Horticulture and Forestry, Bharsar-246123, Pauri Garhwal, Uttarakhand, India \\ ${ }^{2}$ Department of Nematology, CCSHAU, Hisar, Haryana, India \\ ${ }^{3}$ Department of fruit science, VCSGUUHF, Bharsar, Uttrakhand, India \\ *Corresponding author
}

\section{A B S T R A C T}

The correlation and path coefficient analysis were studied in twenty five genotypes in chilli for 15 different qualitative and quantitative characters. Correlation coefficients at

\section{Keywords}

Capsicum annuum

L., Correlation and

Path coefficients

Article Info

Accepted:

04 October 2018

Available Online:

10 November 2018 genotypic and phenotypic levels indicated that fruit yield per plant was positively and significantly correlated with fruit length, plant stem girth, fruit pericarp thickness, average fruit weight, number of fruits per plant, number of primary branches, fruit breadth and plant height but negative and significant association was found with days taken for first flowering and ascorbic acid content indicating that early flowering and early picking might be associated with increasing the fruits yield per plant. Path coefficient analysis revealed that the highest positive direct effect on fruit yield per plant was exerted by average fruit weight followed by fruit length, fruit pericarp thickness, number of fruits per plant, fruit breadth, number of seeds per fruit, days taken for first flowering, number of primary branches, plant height and days to first fruit harvesting, while as highest negative direct effect on fruit yield per plant was exerted by ascorbic acid content, number of branches per plant, plant stem girth and days for $50 \%$ flowering. Therefore, selection should be practiced for average fruit weight, number of fruits per plant, fruit pericarp thickness and fruit length for direct improvement of fruit yield per plant.

\section{Introduction}

Chilli is the universal spice of India. Being an important commercial crop, it finds diverse utilities as a spice, condiment, culinary supplement, medicine, vegetable and ornamental plant. The important states growing chilli are Andhra Pradesh, Orissa, Maharashtra, West Bengal, Karnataka, Rajasthan and Tamil Nadu. Chilli has two important commercial qualities, red colour due to pigment capsanthin and biting pungency attributed by capsaicin. Hence, there is need for development of new varieties and hybrids with high productivity.

Knowledge of inter character relationship is very important in plant breeding for indirect selection for characters that are not easily measured. However, under complex situation, correlation alone become insufficient to explain relationships among characters and 
thus path analysis of economic yield components with yield is important. However in green chilli is meager to the study on correlation and path analysis in chilli for green vegetable yield. Therefore, field investigation was carried out with yield is important. However in green chilli is meager to the study on correlation and path analysis in chilli for green vegetable yield.

Therefore, field investigation was carried out with a view to study the character association and direct and indirect effect of independent characters on dependent green chillli yield by assessing the chilli germplasm stock maintained at Vegetable Research Block of Veer Chandra Singh Garhwali Uttarakhand University of Horticulture and Forestry, Bharsar Campus, Pauri-Garhwal during Kharif 2015.

\section{Materials and Methods}

The present experiment was conducted in the Vegetable Research and Demonstration Block of the Department of Vegetable Science, College of Horticulture, VCSG Uttarakhand University of Horticulture and Forestry, Bharsar, Pauri Garhwal, Uttarakhand. twenty five germplasm lines obtained from different parts of India under completely randomized block design in three replications. Each plot consisted of 12 plants, of which five competitive plants were selected at random for recording the observations. The crop was raised as per the recommended package of practices.

The parameters considered for the plant height (cm), days to first flowering (days), days to $50 \%$ flowering (days), days to first fruit harvesting (days), fruit length $(\mathrm{cm})$, fruit breadth $(\mathrm{cm})$, plant stem girth $(\mathrm{cm})$, pericarp thickness $(\mathrm{mm})$, number of seed per fruit (number), number of primary branches, ascorbic acid content (mg/100g), number of fruits per plant, number of branches, marketable fruit yield per plant $(\mathrm{g})$ and per hectare (q). Ascorbic acid content of fruits will be determined as per the method suggested by Ranganna (1986) using 2, 6-dichlorophenol Indophenol dye.

The phenotypic correlation coefficient and genotypic correlation coefficient and direct and indirect effects were computed by using procedure given by Dewey and Lu (1959).

\section{Results and Discussion}

Knowledge of degree of association of yield with its components is of great importance, because yield is not an independent character, but it is the resultant of the interactions of a number of component characters among themselves as well as with the environment in which the plant grow. Further each character is likely to be modified by action of genes present in the genotypes of plant and also by the environment and it becomes difficult to evaluate this complex character directly.

Therefore, correlation study of yield with its component traits has been executed, to find out the yield contributing traits. The correlation coefficients among the different characters were worked out at phenotypic and genotypic levels. In general, the genotypic correlation coefficients were higher in magnitude than phenotypic correlation coefficients (Table 1 and 2).

The phenotypic correlation coefficients among different characters showed that marketable yield per plant had positive and significant association with fruit length, plant stem girth, fruit pericarp thickness, average fruit weight, number of fruits per plant, number of primary branches, fruit breadth and plant height, while significantly negative correlations were observed with days taken for first flowering and ascorbic acid content, respectively. 
Table.1 Phenotypic and genotypic coefficients of correlation in green chilli yield and component characters

\begin{tabular}{|c|c|c|c|c|c|c|c|c|c|c|c|c|c|c|c|c|}
\hline \multicolumn{2}{|c|}{ Traits } & 1 & 2 & 3 & 4 & 5 & 6 & 7 & 8 & 9 & 10 & 11 & 12 & 13 & 14 & 15 \\
\hline \multirow[t]{2}{*}{1} & $\mathbf{P}$ & 1.00 & $-0.304 * *$ & 0.166 & 0.111 & 0.084 & $-0.236 *$ & 0.064 & $0.402 * *$ & -0.023 & -0.057 & -0.198 & $-0.364 * *$ & 0.089 & 0.104 & $0.294 * *$ \\
\hline & G & 1.00 & $-0.354 * *$ & 0.175 & 0.122 & 0.175 & $-0.347 * *$ & 0.053 & $\mathbf{0 . 5 0 8}^{* *}$ & -0.013 & -0.051 & $-0.242 *$ & $-0.463 * *$ & 0.106 & 0.095 & $0.322 * *$ \\
\hline \multirow[t]{2}{*}{2} & $\mathbf{P}$ & & 1.00 & 0.090 & $0.435 * *$ & -0.051 & 0.019 & -0.010 & $-0.383 * *$ & $0.469 * *$ & 0.053 & -0.163 & $-0.232 *$ & $0.648^{* * *}$ & $-0.353 * *$ & $0.235^{*}$ \\
\hline & G & & 1.00 & 0.105 & -0.079 & -0.079 & -0.007 & 0.038 & $-0.433 * *$ & $0.504 * *$ & 0.051 & -0.165 & $-0.225^{*}$ & $0.679 * *$ & 0.105 & $0.240 *$ \\
\hline \multirow[t]{2}{*}{3} & $\mathbf{P}$ & & & 1.00 & $0.826 * *$ & $-0.524 * *$ & -0.152 & -0.194 & $0.281 *$ & $0.600^{* *}$ & 0.173 & $-0.443 * *$ & $-0.397 * *$ & $-0.557 * *$ & $-0.237 *$ & $0.882 * *$ \\
\hline & G & & & 1.00 & $0.852 * *$ & $-0.743 * *$ & $-0.281 *$ & $-0.302 * *$ & $0.320 * *$ & $0.625^{* *}$ & 0.184 & -0.500 *** & $-0.498 * *$ & $-0.576 * *$ & $-0.261 *$ & $0.898 * *$ \\
\hline \multirow[t]{2}{*}{4} & $\mathbf{P}$ & & & & 1.00 & $-0.381 * *$ & $-0.235^{*}$ & -0.162 & 0.200 & $0.819 * *$ & 0.120 & $-0.445 * *$ & $-0.539 * *$ & $-0.823 * *$ & $-0.300 * *$ & $0.624 * *$ \\
\hline & G & & & & 1.00 & $-0.535 * *$ & $-0.297 * *$ & $-0.237 *$ & 0.220 & $0.848^{* *}$ & 0.140 & $-0.489 * *$ & $-0.667 * *$ & -0.830 *** & $-0.331 * *$ & $0.641 * *$ \\
\hline \multirow[t]{2}{*}{5} & $\mathbf{P}$ & & & & & 1.00 & 0.211 & 0.177 & -0.033 & $-0.234 *$ & -0.109 & $0.392 * *$ & 0.167 & $0.265^{*}$ & $0.293 * *$ & $-0.491^{* *}$ \\
\hline & G & & & & & 1.00 & $0.412 * *$ & $0.681 * *$ & -0.030 & $-0.351 * *$ & -0.154 & $0.584 * *$ & 0.195 & $0.394 * *$ & $0.484 * *$ & $-0.667 * *$ \\
\hline \multirow[t]{2}{*}{6} & $\mathbf{P}$ & & & & & & 1.00 & $0.356 * *$ & -0.097 & -0.165 & 0.130 & 0.213 & 0.196 & 0.069 & 0.157 & -0.177 \\
\hline & G & & & & & & 1.00 & $1.228 * *$ & -0.223 & -0.222 & 0.199 & $0.409 * *$ & $0.384 * *$ & 0.046 & $0.306 * *$ & $-0.269 *$ \\
\hline \multirow[t]{2}{*}{7} & $\mathbf{P}$ & & & & & & & 1.00 & 0.040 & -0.050 & 0.037 & $0.290 *$ & 0.132 & -0.018 & $0.236 *$ & -0.162 \\
\hline & G & & & & & & & 1.00 & 0.127 & -0.097 & 0.060 & $0.727 * *$ & $0.314 * *$ & -0.056 & $0.459 * *$ & $-0.287 *$ \\
\hline \multirow[t]{2}{*}{8} & $\mathbf{P}$ & & & & & & & & 1.00 & $0.313^{* *}$ & -0.084 & 0.000 & $-0.251 *$ & 0.030 & 0.098 & $0.360 * *$ \\
\hline & G & & & & & & & & 1.00 & $0.383^{* * *}$ & -0.084 & 0.005 & $-0.416 * *$ & 0.064 & 0.135 & $0.419 * *$ \\
\hline \multirow[t]{2}{*}{9} & $\mathbf{P}$ & & & & & & & & & 1.00 & -0.067 & $-0.372 * *$ & $-0.512 * *$ & $-0.730^{* * *}$ & $-0.414 * *$ & $0.348^{* * *}$ \\
\hline & G & & & & & & & & & 1.00 & -0.061 & $-0.416 * *$ & $-0.632 * *$ & $-0.750 * *$ & $-0.442 * *$ & $0.356 * *$ \\
\hline \multirow[t]{2}{*}{10} & $\mathbf{P}$ & & & & & & & & & & 1.00 & -0.105 & 0.011 & $-0.251 *$ & 0.145 & 0.222 \\
\hline & G & & & & & & & & & & 1.00 & -0.128 & 0.047 & $-0.269 *$ & 0.151 & $0.226 *$ \\
\hline \multirow[t]{2}{*}{11} & $\mathbf{P}$ & & & & & & & & & & & 1.00 & $0.473 * *$ & $0.344 * *$ & $0.614 * *$ & $0.316^{* *}$ \\
\hline & G & & & & & & & & & & & 1.00 & $0.589 * *$ & $0.379 * *$ & $0.720 * *$ & $0.349 * *$ \\
\hline \multirow[t]{2}{*}{12} & $\mathbf{P}$ & & & & & & & & & & & & 1.00 & $0.434 * *$ & $0.383 * *$ & $-0.249 *$ \\
\hline & G & & & & & & & & & & & & 1.00 & $0.517 * *$ & $0.483 * *$ & $-0.297 * *$ \\
\hline \multirow[t]{2}{*}{13} & $\mathbf{P}$ & & & & & & & & & & & & & 1.00 & $0.269 *$ & $0.227 *$ \\
\hline & G & & & & & & & & & & & & & 1.00 & $0.277^{*}$ & $0.236 *$ \\
\hline \multirow[t]{2}{*}{14} & $\mathbf{P}$ & & & & & & & & & & & & & & 1.00 & -0.062 \\
\hline & G & & & & & & & & & & & & & & 1.00 & -0.078 \\
\hline \multirow[t]{2}{*}{15} & $\mathbf{P}$ & & & & & & & & & & & & & & & 1.00 \\
\hline & G & & & & & & & & & & & & & & & 1.00 \\
\hline
\end{tabular}

*Significant at $5 \%$ level of significance

$* *$ Significant at $1 \%$ level of significance

Where,

1= Plant height $(\mathrm{cm}), 2=$ Fruit breadth $(\mathrm{cm}), 3=$ Fruit length $(\mathrm{cm}), 4=$ Average fruit weight $5=$ Days taken for first flowering, $6=$ Days for 50 per cent flowering,

$7=$ Days to first harvest, $8=$ Plant stem girth $(\mathrm{cm}), 9=$ Fruit pericarp thickness $(\mathrm{mm}), 10=$ Number of seeds per fruit, $11=$ Number of primary branches, $12=$

Ascorbic acid content $(\mathrm{mg} / 100 \mathrm{~g}), 13=$ Number of fruits per plant, 14= Number of branches per plant and 15= Marketable fruit yield per plant $(\mathrm{g})$ 
Table.2 Genotypic and phenotypic path co-efficient analysis for green chilli yield

\begin{tabular}{|c|c|c|c|c|c|c|c|c|c|c|c|c|c|c|c|c|}
\hline \multicolumn{2}{|c|}{ Traits } & 1 & 2 & 3 & 4 & 5 & 6 & 7 & 8 & 9 & 10 & 11 & 12 & 13 & 14 & 15 \\
\hline \multirow[t]{2}{*}{1} & $\mathbf{P}$ & -0.022 & 0.016 & 0.020 & 0.015 & 0.000 & -0.002 & 0.002 & 0.002 & 0.001 & -0.001 & -0.002 & 0.001 & 0.009 & -0.003 & $0.294 * *$ \\
\hline & G & 0.032 & 0.039 & 0.057 & 0.050 & 0.008 & 0.003 & 0.001 & 0.029 & 0.002 & -0.003 & -0.010 & -0.072 & 0.016 & -0.012 & $0.322 * *$ \\
\hline \multirow[t]{2}{*}{2} & $\mathbf{P}$ & 0.007 & 0.053 & 0.011 & 0.060 & 0.000 & 0.000 & 0.000 & -0.002 & -0.015 & 0.001 & -0.002 & 0.001 & -0.064 & 0.009 & $0.235^{*}$ \\
\hline & G & -0.011 & 0.111 & 0.034 & 0.186 & -0.004 & 0.000 & 0.001 & -0.025 & -0.085 & 0.002 & -0.007 & -0.035 & -0.103 & 0.047 & $0.240^{*}$ \\
\hline \multirow[t]{2}{*}{3} & $\mathbf{P}$ & -0.004 & -0.005 & 0.123 & 0.114 & -0.003 & -0.001 & -0.006 & 0.001 & -0.019 & 0.004 & -0.005 & 0.002 & -0.055 & 0.006 & $0.882 * *$ \\
\hline & G & 0.006 & -0.012 & 0.328 & 0.348 & -0.036 & 0.002 & -0.005 & 0.018 & -0.105 & 0.009 & -0.020 & -0.077 & -0.088 & 0.032 & $0.898 * *$ \\
\hline \multirow[t]{2}{*}{4} & $\mathbf{P}$ & -0.002 & -0.023 & 0.102 & 0.138 & -0.002 & -0.002 & -0.005 & 0.001 & -0.025 & 0.003 & -0.005 & 0.002 & -0.081 & 0.008 & $0.624 * *$ \\
\hline & G & 0.004 & -0.051 & 0.279 & 0.409 & -0.026 & 0.002 & -0.004 & 0.012 & -0.143 & 0.007 & -0.019 & -0.103 & -0.126 & 0.041 & $0.641 * *$ \\
\hline \multirow[t]{2}{*}{5} & $\mathbf{P}$ & -0.002 & 0.003 & -0.064 & -0.053 & 0.005 & 0.002 & 0.005 & 0.000 & 0.007 & -0.003 & 0.005 & -0.001 & 0.026 & -0.007 & $-0.491 * *$ \\
\hline & G & 0.006 & 0.009 & -0.244 & -0.219 & 0.048 & -0.003 & 0.012 & -0.002 & 0.059 & -0.008 & 0.023 & 0.030 & 0.060 & -0.060 & $-0.667 * *$ \\
\hline \multirow[t]{2}{*}{6} & $\mathbf{P}$ & 0.005 & -0.001 & -0.019 & -0.032 & 0.001 & -0.008 & 0.010 & 0.000 & 0.005 & 0.003 & 0.003 & -0.001 & 0.007 & -0.004 & -0.177 \\
\hline & G & -0.011 & 0.001 & -0.092 & -0.121 & 0.020 & -0.008 & 0.022 & -0.013 & 0.038 & 0.010 & 0.016 & 0.059 & 0.007 & -0.038 & $-0.269 *$ \\
\hline \multirow[t]{2}{*}{7} & $\mathbf{P}$ & -0.001 & 0.001 & -0.024 & -0.022 & 0.001 & 0.003 & 0.029 & 0.000 & 0.002 & 0.001 & 0.004 & -0.001 & -0.002 & -0.006 & -0.162 \\
\hline & G & 0.002 & -0.004 & -0.099 & -0.097 & 0.033 & -0.010 & 0.018 & 0.007 & 0.016 & 0.003 & 0.029 & 0.049 & -0.009 & -0.057 & $-0.287^{*}$ \\
\hline \multirow[t]{2}{*}{8} & $\mathbf{P}$ & -0.009 & 0.020 & 0.035 & 0.028 & 0.000 & -0.001 & 0.001 & 0.004 & -0.010 & -0.002 & 0.000 & 0.001 & 0.003 & -0.002 & $0.360^{* *}$ \\
\hline & G & 0.016 & 0.048 & 0.105 & 0.090 & -0.001 & 0.002 & 0.002 & -0.057 & -0.065 & -0.004 & 0.000 & -0.064 & 0.010 & -0.017 & $0.419^{* *}$ \\
\hline \multirow[t]{2}{*}{9} & $\mathbf{P}$ & 0.001 & -0.025 & 0.074 & 0.113 & -0.001 & -0.001 & -0.001 & 0.001 & 0.031 & -0.002 & -0.005 & 0.002 & -0.072 & 0.010 & $0.348^{* * *}$ \\
\hline & G & 0.000 & -0.056 & 0.205 & 0.347 & -0.017 & 0.002 & -0.002 & -0.022 & 0.169 & -0.003 & -0.016 & -0.098 & -0.114 & 0.055 & $0.356^{* *}$ \\
\hline \multirow[t]{2}{*}{10} & $\mathbf{P}$ & 0.001 & -0.003 & 0.021 & 0.017 & -0.001 & 0.001 & 0.001 & 0.000 & 0.002 & 0.024 & -0.001 & 0.000 & -0.025 & -0.004 & 0.222 \\
\hline & G & -0.002 & -0.006 & 0.060 & 0.057 & -0.007 & -0.002 & 0.001 & -0.005 & 0.010 & 0.049 & -0.005 & 0.007 & -0.041 & -0.019 & $0.226^{*}$ \\
\hline \multirow[t]{2}{*}{11} & $\mathbf{P}$ & 0.004 & 0.009 & -0.054 & -0.062 & 0.002 & 0.002 & 0.009 & 0.000 & 0.012 & -0.003 & 0.012 & -0.002 & 0.034 & -0.015 & $0.316^{* * *}$ \\
\hline & G & -0.008 & 0.018 & -0.164 & -0.200 & 0.028 & -0.003 & 0.013 & 0.000 & 0.070 & -0.006 & 0.039 & 0.091 & 0.058 & -0.089 & $0.349^{* *}$ \\
\hline \multirow[t]{2}{*}{12} & $\mathbf{P}$ & 0.008 & 0.012 & -0.049 & -0.075 & 0.001 & 0.001 & 0.004 & -0.001 & 0.016 & 0.000 & 0.006 & -0.004 & 0.043 & -0.010 & $-0.249 *$ \\
\hline & G & -0.015 & 0.025 & -0.163 & -0.273 & 0.009 & -0.003 & 0.006 & -0.024 & 0.107 & 0.002 & 0.023 & 0.155 & 0.079 & -0.060 & $-0.297 * *$ \\
\hline \multirow[t]{2}{*}{13} & $\mathbf{P}$ & -0.002 & 0.034 & -0.069 & -0.114 & 0.001 & 0.001 & -0.001 & 0.000 & 0.023 & -0.006 & 0.004 & -0.002 & 0.098 & -0.007 & $-0.227^{*}$ \\
\hline & G & 0.003 & 0.075 & -0.189 & -0.340 & 0.019 & 0.000 & -0.001 & 0.004 & 0.127 & -0.013 & 0.015 & 0.080 & 0.152 & -0.034 & $0.236^{*}$ \\
\hline \multirow[t]{2}{*}{14} & $\mathbf{P}$ & -0.002 & 0.019 & -0.029 & -0.041 & 0.001 & 0.001 & 0.007 & 0.000 & 0.013 & 0.003 & 0.008 & -0.002 & 0.026 & -0.025 & -0.062 \\
\hline & G & 0.003 & 0.042 & -0.086 & -0.135 & 0.023 & -0.002 & 0.008 & 0.008 & 0.075 & 0.007 & 0.028 & 0.075 & 0.042 & -0.124 & -0.078 \\
\hline
\end{tabular}

Where,

1= Plant height $(\mathrm{cm}), 2=$ Fruit breadth $(\mathrm{cm}), 3=$ Fruit length $(\mathrm{cm}), 4=$ Average fruit weight $5=$ Days taken for first flowering, $6=$ Days for $50 \%$ flowering, $7=$

Days to first harvest, $8=$ Plant stem girth $(\mathrm{cm}), 9=$ Fruit pericarp thickness $(\mathrm{mm}), 10=$ Number of seeds per fruit, $11=$ Number of primary branches, $12=$ Ascorbic acid content $(\mathrm{mg} / 100 \mathrm{~g}), 13=$ Number of fruits per plant, 14= Number of branches per plant and 15=Genotypic and Phenotypic correlation coefficient for marketable fruit yield per plant $(\mathrm{g})$

Residual effect $=0.00227$ 
The genotypic correlation coefficients among different characters showed that marketable yield per plant had positive and significant association with fruit length, plant stem girth, fruit pericarp thickness, average fruit weight, number of fruits per plant, plant height, fruit breadth, days for $50 \%$ flowering, number of primary branches and number of seeds per fruit. While significantly negative correlations were observed with days taken for first flowering, days to first harvest and ascorbic acid content, respectively. Beside this, fruit length resulted in positive and significant association with average, fruit weight, Fruit pericarp thickness, Plant stem girth and it revealed significantly negative correlation with days taken to first flowering, number of primary branches, ascorbic acid content and number of branches per plant, respectively. Significantly positive correlation of average fruit weight was found with fruit pericarp thickness, while significant negative association of this trait was found with days taken to first flowering, days for $50 \%$ flowering and ascorbic acid content. In the mean while plant height resulted in positive and significant association with plant stem girth, while significant negative association of this trait was found with ascorbic acid content, fruit breadth and days for 50\% flowering, respectively. In the meanwhile, plant stem girth was significantly and positively correlated with fruit pericarp thickness, while significant negative correlation of this trait was found with ascorbic acid content. Fruit breadth showed significantly positive correlation with number of fruit per plant and fruit pericarp thickness, while negative association of this trait was observed with plant stem girth and ascorbic acid content. Number of primary branches revealed significantly positive correlation with ascorbic acid content, number of fruit per plant and number of branches per plant. In the mean while ascorbic acid content resulted in positive and significant association with number of fruit per plant and number of branches per plant. Number of fruit per plant showed significantly positive correlation with number of branches per plant. Similar correlation of yield with various horticultural and quality traits have also been reported earlier by several workers viz., Bijalwan and Mishra (2016), Hasan et al., (2016), Aklilu et al., (2016) and Janaki et al., (2016).

Although correlation studies are helpful in determining the components of yield but it does not provide a clear picture of nature and extent of contributions made by number of independent traits. Path coefficient analysis devised by Dewey and Lu (1959), however, provides a realistic basis for allocation of appropriate weight age to various attributes while designing a pragmatic programme for the improvement of yield. Path coefficient analysis depicts the effects of different independent characters individually and in combination with other characters on the expression of different characters on marketable fruit yield per plant.

The path coefficient analysis at genotypic level revealed that marketable yield per plant has maximum positive direct effect on that average fruit weight has maximum positive direct effect on marketable fruit yield per plant followed by fruit length, fruit pericarp thickness, number of fruits per plant, fruit breadth, number of seeds per fruit, days taken for first flowering, number of primary branches, plant height and days to first fruit harvesting. While, negative direct effect of ascorbic acid content, number of branches per plant, plant stem girth and days for $50 \%$ flowering was observed on marketable fruit yield per plant. Maximum positive indirect effects of average fruit weight via fruit length, fruit pericarp thickness via average fruit weight, average fruit weight via number of fruits per plant, fruit length via average fruit weight, fruit length via fruit pericarp 
thickness, average fruit weight via number of primary branches, Fruit pericarp thickness via number of fruits per plant, Fruit pericarp thickness via ascorbic acid content, Fruit length via plant stem girth, Fruit breadth via number of fruits per plant and Fruit breadth via fruit pericarp thickness was observed on marketable fruit yield per plant. Chattopadhyay et al., (2011), Diwaker et al., (2012), Patel et al., (2015), Bijalwan and Mishra (2016), Hasan et al., (2016) and Janaki et al., (2016) had reported similar direct and indirect effects of various horticultural and quality traits on yield in chilli.

\section{References}

Aklilu S, Abebie B, Wogari D and Adeferis T. 2016. Genetic variability and association of characters in Ethiopian hot pepper (Capsicum annum L.) Landraces. Journal of Agricultural Sciences 1(61): 19-36.

Bijalwan P and Mishra A C. 2016. Correlation and Path Coefficient Analysis in Chilli (Capsicum annuum L.) for Yield and Yield Attributing Traits. International Journal of Science and Research 5(2): 1588- 1592

Chattopadhyay A, Sharangi A B, Dai N and Dutta N. 2011. Diversity of genetic resources and genetic association analyses of green and dry chillies of Eastern India. Chilean Journal of Agriculture Research (71): 350-356.
Dewey J R and Lu K H. 1959. A correlation and path coefficient analysis of components of crested wheat grass seed production. Agronomy Journal 51: 515518.

Diwaker K, Bahadur V, Rangare S B and Singh D. 2012. Genetic variability, heritability and correlation studies in chilli (Capsicum annuum L.). Horticulture Flora Research Spectrum 1(3): 248-252.

Hasan R, Akand M, Alam N, Bashar A and Mahmudul Huque A K M. 2016. Genetic Association Analysis and Selection Indices for Yield Attributing Traits in Available Chilli (Capsicum annuum L.) Genotypes. Molecular Plant Breeding 19(7): 1-9

Janaki M, Naidu L N, Ramana C V and Rao M P. 2016. Character association and path analysis studies of quantitative traits in chilli (Capsicum annuum L.). Environment and Ecology 34(2): 698702

Patel D K, Patel B R, Patel J R and Kuchhadiya G V. 2015. Genetic variability and character association studies for green fruit yield and quality component traits in chilli (Capsicum annuum var. longum (dc.) sendt.). Electronic Journal of Plant Breeding 6(2): 472-478.

Ranganna S. 1986. Handbook of analysis and quality control for fruit and vegetable products. $2^{\text {nd }}$ ed. Tata McGraw Hill, New Delhi. pp. 105-106.

\section{How to cite this article:}

Manoj Kumar Bundela, S.C. Pant, Madhuri and Kulveer Singh. 2018. Correlation and Path Coefficient Analysis in Chilli (Capsicum annuum L.) for Yield and Yield Attributing Traits. Int.J.Curr.Microbiol.App.Sci. 7(11): 77-82. doi: https://doi.org/10.20546/ijcmas.2018.711.011 